\title{
On discriminating between geometric strategies of surface-based orientation
}

\author{
Bradley R. Sturz * and Kent D. Bodily \\ Department of Psychology, Georgia Southern University, Statesboro, GA, USA
}

Edited by:

Ken Cheng, Macquarie University, Australia

Reviewed by:

Ken Cheng, Macquarie University, Australia

\section{*Correspondence:}

Bradley R. Sturz, Department of Psychology, Georgia Southern

University, P.O. Box 8041, Statesboro, GA 30460, USA.

e-mail: bradleysturz@ georgiasouthern.edu
Recently, a debate has manifested in the spatial learning literature regarding the shape parameters by which mobile organisms orient with respect to the environment. On one hand are principal-axis-based strategies which suggest that organisms extract the major and minor principal axes of space which pass through the centroid and approximate length and width of the entire space, respectively. On the other hand are medial-axis-based strategies which suggest that organisms extract a trunk-and-branch system similar to the skeleton of a shape. With competing explanations comes the necessity to devise experiments capable of producing divergent predictions. Here, we suggest that a recent experiment (i.e., Sturz and Bodily, 2011a) may be able to shed empirical light on this debate. Specifically, we suggest that a reevaluation of the design reveals that the enclosures used for training and testing appear to produce divergent predictions between these strategies. We suggest that the obtained data appear inconsistent with a medial-axis-based strategy and that the study may provide an example of the types of designs capable of discriminating between these geometric strategies of surface-based orientation. Such an approach appears critical to fundamental issues regarding the nature of space and spatial perception.

Keywords: orientation, geometric strategies, medial axis, principal axis
Many mobile organisms that are trained to locate a distinct visual cue in a corner of a rectangular enclosure will respond to an equivalent (above chance) extent to both the trained corner and its $180^{\circ}$ rotational equivalent (e.g., corners A and B in Figure 1) when the distinct visual cue is removed or rendered useless during subsequent testing (for a review, see Cheng and Newcombe, 2005). This "rotational error" phenomenon has been taken as evidence that organisms incidentally encode information about environmental geometry (Cheng, 1986; Gallistel, 1990).

Recently, a debate has manifested in the spatial learning literature regarding the shape parameters by which mobile organisms orient with respect to the environment (Kelly et al., 2011a,b; Sturz and Bodily, 2011b). On the one hand are principal-axis-based strategies which propose that organisms extract the major and minor principal axes of space. The major and minor principal axes pass through the centroid and approximate length and width of the entire space, respectively (for a detailed mathematical and mechanical definition, see Cheng, 2005; see also Cheng and Gallistel, 2005; Bodily et al., 2011; Sturz et al., 2011; left panel, Figure 1). On the other hand are medial-axis-based strategies which propose that organisms extract a trunk-and-branch system similar to the skeleton of a shape (see Blum, 1967; Cheng, 2005; Kelly and Durocher, 2011; Kelly et al., 2011a,b; see right panel, Figure 1).

Although the parameters underlying these orientation strategies are geometrically independent, both strategies have been proposed in an effort to account for the ubiquitous rotational error phenomenon described above (for a review, see Cheng, 2005). According to a principal-axis-based strategy (Figure 1, left panel), when organisms learn to approach a distinct cue at location A, they also incidentally learn to search at the right-hand side of the major principal axis of space (and/or the left-hand side of the minor principal axis of space). When tested in the absence of the distinct cue, a strategy of searching at the right-hand side of the major principal axis (and/or the left-hand side of the minor principal axis) results in equivalent responses to location $\mathrm{A}$ and location B (i.e., the rotational error). In contrast, according to a medial-axis-based strategy, when organisms learn to approach a distinct cue at location $\mathrm{A}$, they also incidentally learn to search at the end of the trunk's terminal right branch. When tested in the absence of the distinct cue, a strategy of searching at the end of the trunk's terminal right branch results in equivalent responses to location A and location B (i.e., the rotational error).

Given these apparent competing explanations of surface-based orientation, it seems critical to devise experiments that are capable of producing divergent predictions - predictions that allow for the discrimination between principal-axis and medial-axis-based strategies of surface-based orientation (see Kelly and Durocher, 2011). In what follows, we reevaluate a recent experiment on geometric orientation strategies (i.e., Sturz and Bodily, 2011a). We suggest that this reevaluation offers a potential example of the types of designs that may be capable of producing divergent predictions between principal-axis and medial-axis-based strategies designs that may shed empirical light on the this theoretical debate.

Recently, we (Sturz and Bodily, 2011a) trained two groups of human participants in a dynamic, three-dimensional virtual environment to respond to two particular corners in hexagon-shaped enclosures [Top Right (TR) and Bottom Left (BL); see Figure 2]. The use of the hexagon-shaped enclosures allowed us to eliminate local geometric cues such as wall lengths and corner angles and isolate global geometric cues for orientation. Importantly, these 


\section{Principal Axis}

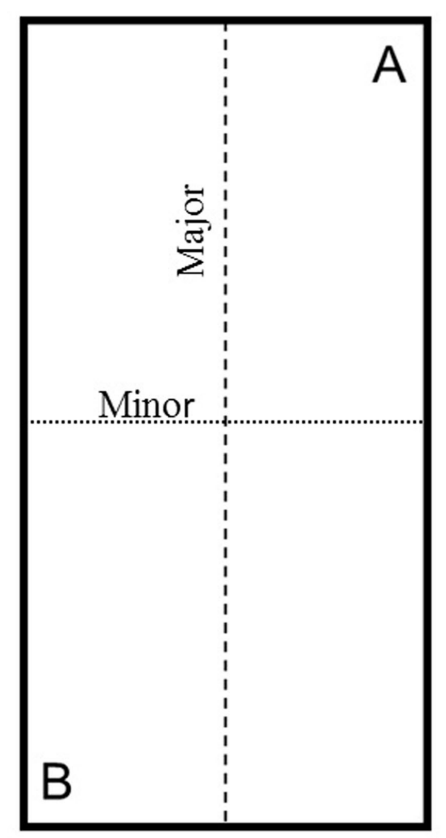

\section{Medial Axis}

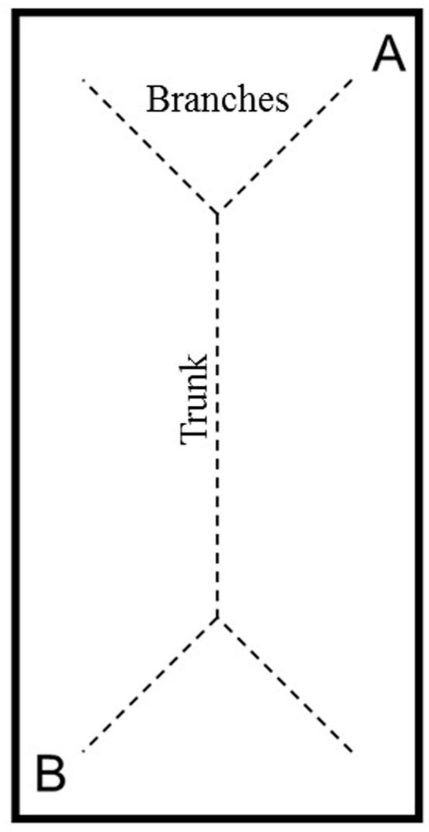

FIGURE 1 | Illustration of principal-axis-based (left panel) and medial-axis-based (right panel) strategies. A represents trained location, and B represents the rotationally equivalent location.

hexagon-shaped enclosures provided participants with enclosures that differed with respect to a hypothetical discriminability ratio between the major and minor principal axes of space. Our rationale was that the discriminability of the principal axis of space (and hence its usefulness as an orienting cue) may be a function of its proportional relationship to the minor principal axis of space. Specifically, we hypothesized that an ability to utilize the major principal axis of space as an orienting cue should increase as it becomes more discriminable from the minor principal axis of space. To that end, we selected two training enclosures for which the major principal axis should be more discriminable from the minor principal axis for one of the enclosures relative to the other (i.e., 0.75 versus 0.25 ; refer to Figure 2). Subsequent testing in the trained and novel-shaped enclosures revealed that participants trained in the enclosure with the major principal axis hypothesized to be more discriminable (i.e., 0.75 ) allocated relatively more responses to locations specified by the trained side of the major principal axis of space. We interpreted this obtained difference between the groups as evidence that the discriminability of the major principal axis of space relative to the minor principal axis of space influenced its usefulness as an orienting cue.

Although we did not design this experiment to produce divergent predictions between principal-axis and medial-axis-based strategies of surface-based orientation, we suggest that a reevaluation of the design produces unique predictions for each geometric strategy. Specifically, we calculated the medial axis of space for each training and testing enclosure of our experiment (see Figure 3). It is important to note that despite the difference in the enclosure shapes experienced during training by each group, the trunk lengths and terminal-branch lengths were identical in both enclosures. In other words, despite our manipulation of enclosure shape, both groups experienced equivalent training with respect to the medial axis of space. If participants had extracted the medial axis of space during training, then participants in both groups would have been rewarded for searching at the end of the trunk's terminal right branch. As a result, participants in both groups should have learned an identical medialaxis-based orientation strategy. Consequently, an application of this learned medial-axis-based strategy during the subsequent testing should have resulted in equivalent performance across groups.

In short, we suggest that under the conditions of our experiment, a medial-axis-based strategy would predict no difference between the groups during testing because both groups would have relied on identical trunk lengths and terminal-branch lengths. However, obtained results indicated that the groups performed differently during testing. We suggest that such a result appears inconsistent with a medial-axis-based strategy of surface-based orientation.

We acknowledge that this retrospective interpretation of our experiment as outlined above is by no means definitive. Specifically, we acknowledge that this interpretation is constrained by numerous differences in potentially critical variables between orientation experiments such as variations in training and testing shapes and/or differences in the species under investigation. In addition, we acknowledge the limitations of basing theoretical 


\section{Group 0.75}

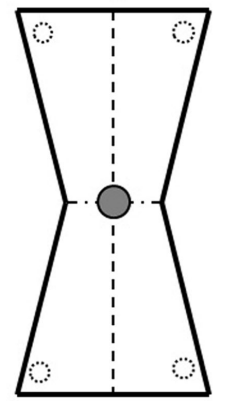

\section{Training}

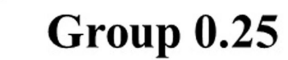

\section{Testing}
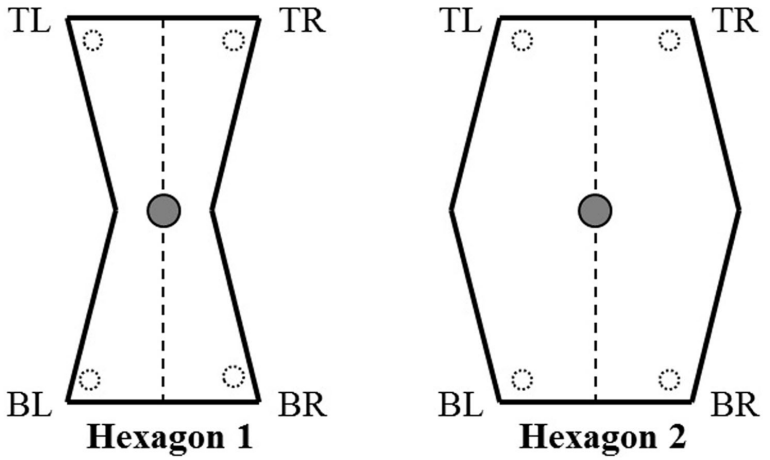

Hexagon 2

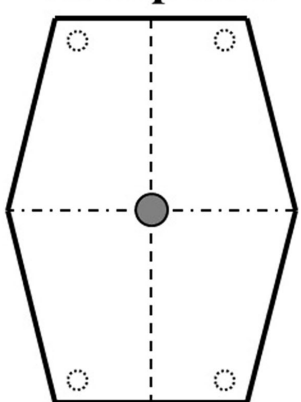

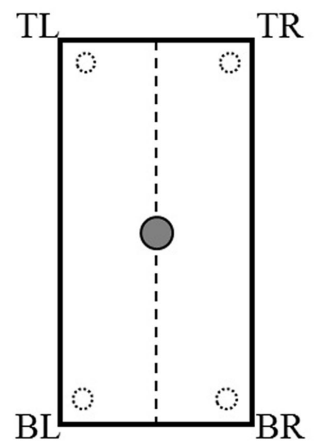

Rectangle
$\mathrm{TL}$

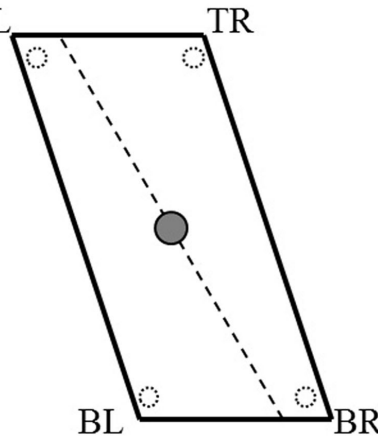

Parallelogram 1

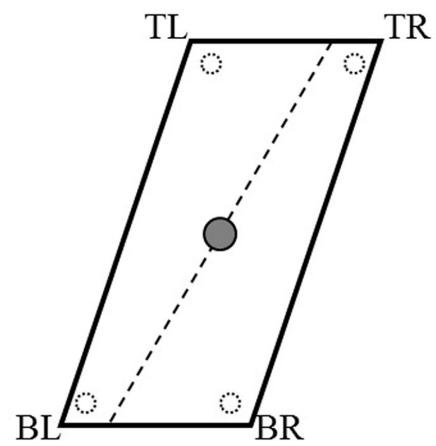

Parallelogram 2

\footnotetext{
$\mathrm{TL}=$ Top Left $\quad \mathrm{TR}=$ Top Right $\quad \mathrm{BL}=$ Bottom Left $\quad \mathrm{BR}=$ Bottom Right

i=Response Locations $\quad \vdots$ = Major Principal Axis $\quad \vdots$ = Minor Principal Axis $\mathrm{O}=$ Start Locations
}

FIGURE 2 | Schematics of training and testing enclosures of Sturz and Bodily (2011a). Unfilled circles represent invisible response locations, and filled circles represent start locations.

conclusions on statistically null empirical results (as predicted by a medial-axis-based strategy in the present case); however, this statistical limitation could be remedied by recent efforts to utilize Bayesian analyses for determining whether a null hypothesis is supported by the data (see Gallistel, 2009). Despite these limitations, the interpretation provided here may serve as an example of the types of investigations that could discriminate between these strategies of surface-based orientation and may serve as an impetus for adopting a converging evidence approach to illuminating the shape parameters by which mobile organisms orient with respect to the environment.

Such an approach seems especially critical to remaining issues in comparative spatial cognition regarding perceptible space (i.e., entire space is viewable from a single vantage point) and imperceptible space (i.e., entire space is not viewable from a single vantage point), and it remains unclear the extent to which principalaxis and medial-axis-based strategies are able to account for this kind of partitioning of space into subspaces. Overall, these 

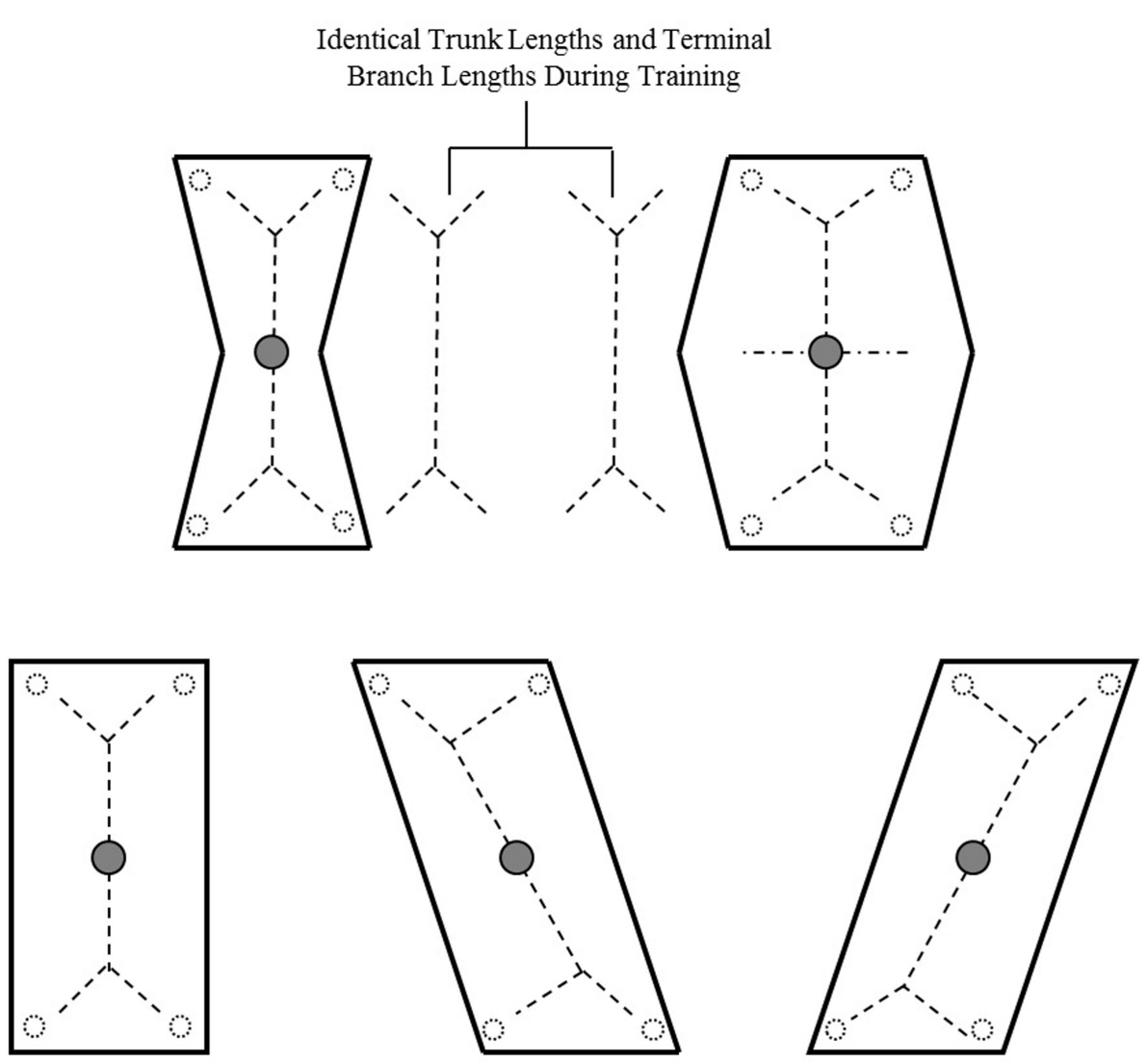

i=Response Locations $\quad$ Medial Axis $\quad \bigcirc=$ Start Locations

FIGURE 3 | Schematics of training and testing enclosures of Sturz and Bodily (2011a) with the medial axis for each enclosure. Unfilled circles represent invisible response locations, and filled circles represent start locations.

issues appear to reflect larger issues of our objective definitions of space and organisms' subjective experiences of that space. Collectively, these issues appear to reflect fundamental and unanswered psychophysical questions regarding the nature of space and spatial perception.

\section{REFERENCES}

Blum, H. (1967). "A transformation for extracting new descriptors of shape," in Models for the Perception of Speech and Visual Form, ed. W. WathenDunn (Cambridge, MA: MIT Press), 362-380.

Bodily, K. D., Eastman, C. K., and Sturz, B. R. (2011). Neither by global nor local cues alone: evidence for a unified orientation process. Anim. Cogn. 14, 665-674.

Cheng, K. (1986). A purely geometric module in the rat's spatial representation. Cognition 23, 149-178.
Cheng, K. (2005). Reflections on geometry and navigation. Conn. Sci. 17, 5-21.

Cheng, K., and Gallistel, C. R. (2005). Shape parameters explain data from spatial transformations: comment on Pearce et al. (2004) and Tommasi and Polli (2004). J. Exp. Psychol. Anim. Behav. Process. 31, 254-259.

Cheng, K., and Newcombe, N. S. (2005). Is there a geometric module for spatial orientation? Squaring theory and evidence. Psychon. Bull. Rev. 12, $1-23$.

\section{ACKNOWLEDGMENTS}

The preparation of this manuscript was supported in part by funds from the Office of the Vice President for Research and the Jack N. Averitt College of Graduate Studies at Georgia Southern University.

Gallistel, C. R. (1990). The Organization of Learning. Cambridge, MA: MIT Press.

Gallistel, C. R. (2009). The importance of proving the null. Psychol. Rev. 116, 439-453.

Kelly, D. M., Chiandetti, C., and Vallortigara, G. (2011a). Re-orienting in space: do animals use global or local geometry strategies? Biol. Lett. 7, 372-375.

Kelly, D. M., Durocher, S., Chiandetti, C., and Vallortigara, G. (2011b). A misunderstanding of principal and medial axes? Reply to Sturz and Bodily. Biol. Lett. 7, 649-650.
Kelly, D. M., and Durocher, S. (2011). Comparing geometric models for orientation: medial vs. principal axes. Commun. Integr. Biol. 4, 710-712.

Sturz, B. R., and Bodily, K. D. (2011a). Is surface-based orientation influenced by a proportional relationship of shape parameters? Psychon. Bull. Rev. 18, 848-854.

Sturz, B. R., and Bodily, K. D. (2011b). Of global space or perceived place? Comment on Kelly et al. Biol. Lett. 7, 647-648.

Sturz, B. R., Gurley, T., and Bodily, K. D. (2011). Orientation in 
trapezoid-shaped enclosures: implications for theoretical accounts of geometry learning. J. Exp. Psychol. Anim. Behav. Process. 37, 246-253.

Conflict of Interest Statement: The authors declare that the research was conducted in the absence of any commercial or financial relationships that could be construed as a potential conflict of interest.

Received: 21 March 2012; accepted: 29 March 2012; published online: 25 April 2012.
Citation: Sturz BR and Bodily KD (2012) On discriminating between geometric strategies of surface-based orientation. Front. Psychology 3:112. doi: 10.3389/fpsyg.2012.00112

This article was submitted to Frontiers in Comparative Psychology, a specialty of Frontiers in Psychology.
Copyright (C) 2012 Sturz and Bodily. This is an open-access article distributed under the terms of the Creative Commons Attribution Non Commercial License, which permits non-commercial use, distribution, and reproduction in other forums, provided the original authors and source are credited. 